\title{
UNA NUEVA ESPECIE DEL GÉNERO SALVIA SECCIÓN MEMBRANACEAE DE GUERRERO, MÉXICO
}

\author{
Itzi Fragoso-MartíneZ ${ }^{1,2}$ y Martha MartíneZ-Gordillo ${ }^{1}$ \\ ${ }^{1}$ Universidad Nacional Autónoma de México, Herbario de la Facultad de Ciencias, \\ Apdo. postal 70-399, 04510 México, D.F., México. \\ 2Autor para la correspondencia: itzi@ciencias.unam.mx
}

\section{RESUMEN}

Se describe e ilustra una nueva especie de Salvia sección Membranaceae: Salvia confertispicata, la cual pertenece a la subsección Lophanthoideae y comparte semejanzas morfológicas con Salvia mocinoi, pero difiere de la misma por presentar inflorescencias compactas, brácteas rómbicas, ascendentes, con ápice caudado y base cuneada, cáliz glabrado en la antesis, tubo de la corola invaginado y más largo que en todas las especies de la subsección Lophanthoideae. Se trata de una especie que ha sido recolectada solo en el estado de Guerrero, en donde prospera en bosques de Quercus, bosques de Pinus-Quercus, bosques de Pinus, bosques mesófilos de montaña y bosques tropicales caducifolios.

Palabras clave: Calosphace, Guerrero, Lamiaceae, Membranaceae, México, Salvia.

\begin{abstract}
A new species of Salvia section Membranaceae is described and illustrated: Salvia confertispicata belongs to subsection Lophanthoideae and shares morphological similarities with Salvia mocinoi; nevertheless, the former differs in having compact inflorescences, rhombic ascendant bracts with caudate apex and cuneate base, glabrate calyx at anthesis, an invaginated corolla tube which is the longest of all the species in subsection Lophanthoideae. The new species had only been collected in the state of Guerrero, where it is commonly found in oak forests, pine-oak forests, pine forests, cloud forests, and tropical deciduous forests.
\end{abstract}

Key words: Calosphace, Guerrero, Lamiaceae, Membranaceae, Mexico, Salvia. 
La familia Lamiaceae es una de las más diversas de México, ocupando el octavo lugar en cuanto a riqueza de especies (Villaseñor, 2003). En ella destaca el género Salvia L., segundo por su diversidad y endemismo en el país, con alrededor de 300 especies (Villaseñor, 2004) y un endemismo de ca. 88\% (Ramamoorthy y Elliot, 1998). Se trata de un taxon con afinidad por las zonas templadas, cuyos representantes crecen principalmente en tipos de vegetación como bosques de coníferas, de Quercus y mesófilos de montaña, y presenta una mayor concentración de especies en Oaxaca, Puebla, Guerrero, Michoacán y Jalisco (Ramamoorthy y Elliot, 1998). La sección Membranaceae, propuesta por Bentham (1833), incluye 7-14 especies, distribuidas principalmente en México, tres de ellas en Mesoamérica (Epling, 1939; Epling, 1940; Fragoso-Martínez, 2011; Klitgaard, 2012), en su mayoría se encuentran al sur del Eje Neovolcánico en los estados de Guerrero y Oaxaca, prefiriendo los bosques de Quercus. Esta sección se reconoce por presentar brácteas grandes, persistentes, venoso-reticuladas, con frecuencia de colores vistosos, corola con el labio anterior más largo que el superior y los estambres insertos en la corola. Se divide en dos subsecciones: Lophanthoideae Epling y Elschotzioideae Epling, la primera con 8-10 especies y la segunda con cuatro (Fragoso-Martínez, 2011).

Como parte del trabajo de revisión taxonómica de la sección Membranaceae en la República Mexicana, se detectaron en el estado de Guerrero plantas con un conjunto de características diferentes al resto de las especies de la subsección Lophanthoideae, tales como: las inflorescencias compactas con brácteas rómbicas, ascendentes, con el ápice caudado y la base cuneada, el cáliz glabrado, el tubo de la corola invaginado y más largo que en todas las otras especies de la subsección, por lo que aquí proponemos como una especie nueva para la ciencia a:

Salvia confertispicata I. Fragoso et Mart. Gord. sp. nov. (Fig. 1).

Salviae mocinoi Benth. affinis, sed inflorescentiis compactis, bracteis rhombeis, caudatis apice, atenuatis base, ascendentibus, calyce glabrato, tubo corollae longiore et invaginato.

Sufrútices escandentes, de 0.5-2.5 m de alto, tallo glabro, los entrenudos de 1.5-10 cm de largo, más aproximados entre sí en la parte superior. Hojas con pecíolos acanalados, hírtulos en el canal, de 0.5-3 cm de largo; láminas ovadas, de 2-6.5(-7.5) x 1-3.5(-4) cm, ápice acuminado, margen serrado, con 4-8 dientes por cm, base redondeada, haz glabrescente, escasamente hírtulo en las venas, envés más pálido, en ocasiones con tonos purpúreos, glabrescente, hirsuto en las venas. Inflorescencias en 


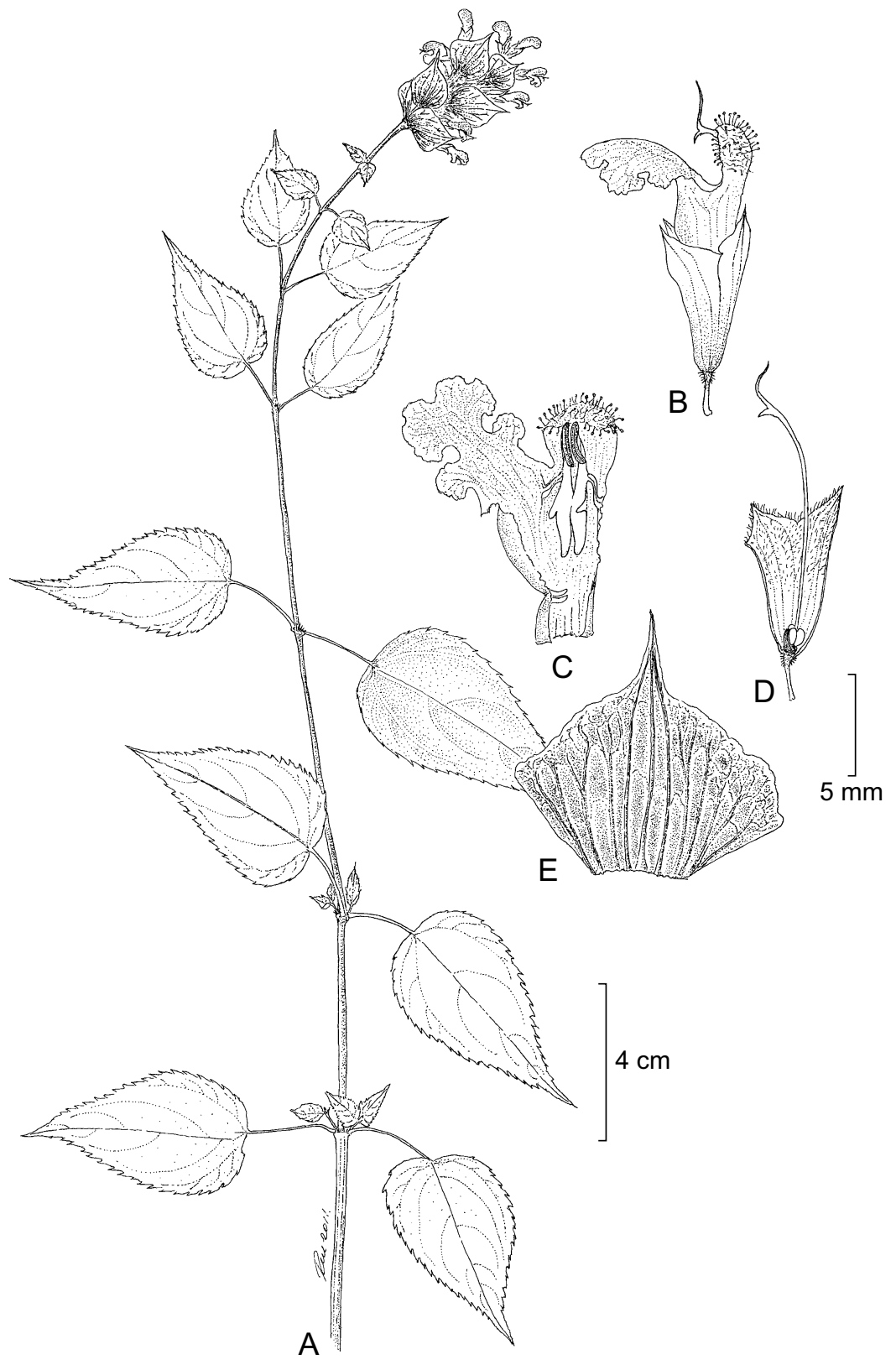

Fig. 1. Salvia confertispicata I. Fragoso et Mart.Gord. A. rama con inflorescencia y hojas; B. flor; C. corte longitudinal de la corola mostrando el androceo; D. corte longitudinal del cáliz, mostrando el estilo y las núculas; E. bráctea extendida. (A dibujado de Castillo 16 (FCME); B-E de Fonseca 117 (FCME)). 
espigas terminales, compactas, de 1-4(-6.5) cm de largo, pedúnculos de 1.5-4(-6.5) $\mathrm{cm}$ de largo, verticilastros distanciados entre sí 0.3-1 cm; brácteas rómbicas, ascendentes, rojizas o purpúreas, foliáceas, de igual tamaño que los cálices, de 0.9-1.5(-1.9) x 0.9-2 cm, ápice caudado, margen repando, ciliado, base cuneada, gruesas, opacas, corrugadas. Flores 5-15 por verticilastro, subsésiles, pedicelos erectos, de 1-3 mm de largo; cáliz purpúreo, tubo de 6-8 $\mathrm{mm}$ de largo, dientes acuminados, el posterior ligeramente más largo que los anteriores y con 5 venas gruesas y 2 delgadas (3-nervio en el ápice), de 1-4 mm de largo, glabrescente; corola de color violeta o morada, tubo ventricoso, de 6-12(-16) mm de largo, más grande que el cáliz, invaginado, la superficie interna en la parte proximal, generalmente con 2 papilas deltoides grandes, centrales y aproximadas, en ocasiones una tercer papila pequeña y hemicíclica en posición superior a las otras, la parte distal con 2 estaminodios pequeños, horizontales, cercanos a la garganta; labio posterior formando una galea ciliado-glandular, de 3-5(-6) $\mathrm{mm}$ de largo; labio anterior de 5-10 $\mathrm{mm}$ de largo; gubernáculo de 3-5 $\mathrm{mm}$ de largo, dorso con tricomas glandulares, diminutos; estilo de (0.8-)1-1.5 cm de largo, glabro, con la rama anterior no excavada, más pequeña, la posterior de 1-3 $\mathrm{mm}$ de largo. Núculas elípticas, lisas, pardas, de $1.5 \mathrm{~mm}$ de largo.

Tipo: México, Guerrero: Municipio: Chilpancingo, $4.5 \mathrm{~km}$ al SW de Omiltemi por el camino a La Soledad, 2360 m, 18-enero-1984, J. Contreras 1456 (holotipo: FCME; isotipos: IEB, MEXU, NY).

Distribución. Salvia confertispicata se conoce por el momento del estado de Guerrero (Fig. 2). Ha sido recolectada en altitudes que van de 920 a $2850 \mathrm{~m}$, en bosques de Quercus, bosques de Pinus-Quercus, bosques de Pinus, bosques mesófilos de montaña y bosques tropicales caducifolios.

Fenología. Con flores todo el año.

Etimología. El nombre se refiere a la característica de las inflorescencias de la especie, que son compactas.

Ejemplares examinados. MÉXICO. GUERRERO, municipio de Atoyac de Álvarez, a 1100 m al NW del poblado El Paraíso, 920 m, 29 Ago 1986, Ortiz 186 (FCME). Municipio de Chichihualco, $9 \mathrm{~km}$ delante de Carrizal de Bravos, por la carretera a Puerto del Gallo, 2600 m, 20 Feb 1981, Fonseca 117 (FCME). 12 km al SW de Filo de Caballo, camino a Puerto del Gallo, 2850 m, 25 Feb 1984, Martínez 


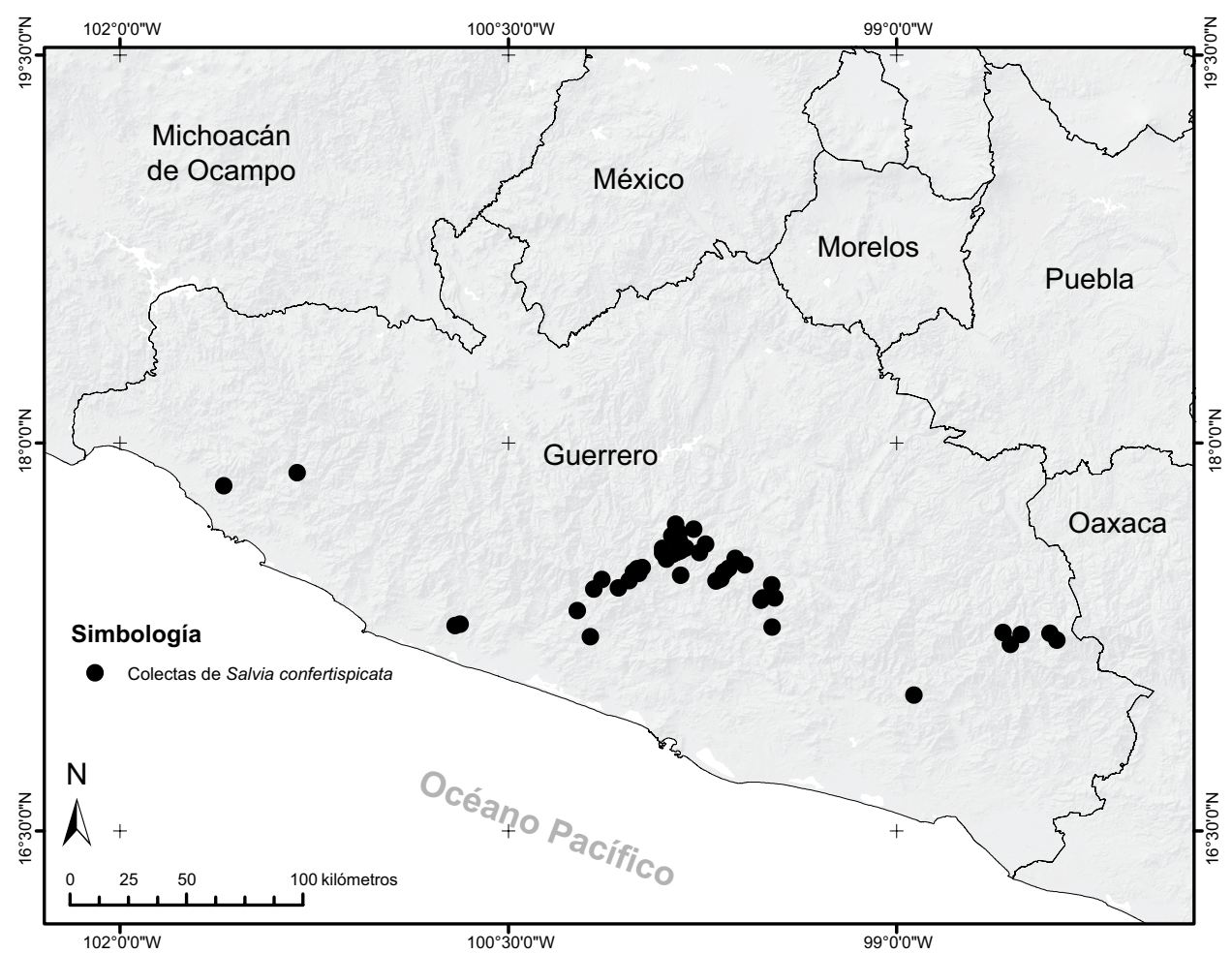

Fig. 2 . Distribución conocida de Salvia confertispicata I. Fragoso et Mart.-Gord.

et al. 6144 (MEXU, XAL). Municipio de Chilpancingo, cerro Alquitrán (cima), 2400 m, 2 May 1969, Kruse 2485 (FCME). El Chayotal, parque estatal Omiltemi, 2400 m, 20 Feb 1994, Diego 6977 (FCME). A 6 km al W de Omiltemi, camino a Soledad las Joyas, 27 Mar 1982, Martínez \& Téllez 270 (ENCB, MEXU, XAL). 3 km al W del caserío del parque estatal de Omiltemi, 2150 m, 9 Jul 1984, Soto-Arenas 1158 (FCME). A $3.5 \mathrm{~km}$ al W de Omiltemi, por el camino que va a la laguna de Agua Fría, 2450 m, 3 Abr 1985, Torres \& R. González 1797bis (FCME). Rincón de la Vía, 15 Dic 1968, Kruse 2329 (FCME). Municipio de General Heliodoro Castillo, a $12 \mathrm{~km}$ de Yextla, cerca de Filo de Caballos, 14 Mar 2002, Castillo 16 (FCME). Camino Carrizal de Bravo, adelante de la desviación a Chichihualco, $2430 \mathrm{~m}, 30$ Jun 2008, Lozada \& Rojas 3454 (FCME). El Jilguero, 2 km al N, 2610 m, 16 Ene 1999, R. Cruz 3547 (FCME, MEXU). Los Bajos, 2550 m, 27 Feb 1998, Diego et al. 7636 (FCME). Municipio de Leonardo Bravo, Los Cajones, 2560 m, 27 Ene 2006, 
Jiménez 1 (FCME). Balsamar, 6 km al NE de Filo de Caballo, 2400 m, 9 Nov 1997, Calónico 6765 (FCME), 6766 (FCME). 4 km adelante de Carrizal de Bravo, hacia Chichihualco, 2390 m, 14 Feb 1982, Campos 301 (FCME). El Carrizal, 2700 m, 17 Abr 2008, Cipriano 1538 (FCME), 17 Abr 2008, M. Cruz 5689 (FCME). Carrizal de Bravo, $1.3 \mathrm{~km}$ al S, 2370 m, 3 Abr 1997, Calónico 1259 (FCME). Carrizal de Bravo, 4 km al S, 2600 m, 4 Mar 1998, Calónico 8298 (FCME). Carrizal de Bravo, $8 \mathrm{~km}$ al SW, Valencia 1278 (CHAP, FCME). Carrizal de Bravo, $2.5 \mathrm{~km}$ al SSE, camino a Chichihualco, barranca al S de cerro Santiago (estanque de Las Truchas), 2441 m, 13 Dic 2001, Reyes et al. 122 (FCME). Filo de Caballos, 2540 m, 17 Abr 2008, Ocampo 5778 (FCME). Filo de Caballo, 7.56 km al S, 2400 m, 29 Mar 1998, Calónico 8518 (FCME). Las Pastillas, $0.5 \mathrm{~km}$ adelante de La Felicidad, rumbo a Chichihualco, 2400 m, 25 Feb 1999, Fonseca 2680 (FCME). Puerto Soleares, 7 km después de Carrizal, hacia Atoyac, 2500 m, 5 Nov 1998, Fonseca 2557 (FCME). Puerto Soleares, $7 \mathrm{~km}$ después de Carrizal, hacia Atoyac, al NW del Asoleadero, 2480 m, 10 Mar 1998, Huerta 197 (FCME). Al N de Puerto Soleares, 2600 m, 3 Dic 1997, Cervantes 184 (FCME). Puerto Soleares, 7 km después de Carrizal, hacia Atoyac, cerro Cacho de Oro, 2550 m, 11 Mar 1998, Fonseca 2438 (FCME). Tres Caminos, $3 \mathrm{~km}$ al adelante del poblado, $2440 \mathrm{~m}, 28$ Oct 1997, B. González 1508 (FCME). Tres Caminos, 15.5 km al SW, 2030 m, 28 Abr 1998, Calónico 8439 (CHAP, FCME). Tres Caminos, 0.75 km al SW, 2400 m, 27 Jun 1997, R. Cruz 1168 (FCME). Municipio de Malinaltepec, cerro de La Luana, 2640 m, 4 Nov 1990, Toledo \& Landa 777 (FCME). Municipio Metlatonoc, km 5 del camino Metlatonoc a Hexoapa, 2150 m, 1 Mar 1984, Lorea 2956 (FCME). Adelante de San Lucas hacia la carr. a Marquelia, 2711 m, 21 Nov 2004, de Santiago \& Diego 1371 (FCME). Sin municipio, Tres Cruces, 2100 m, Jul 1999, Ramírez 23 (FCME). Puerto Pichones, $72 \mathrm{~km}$ al E de Chilpancingo por la carretera Chichihualco-Filo de Caballo, $2450 \mathrm{~m}$, 31 May 1980, Contreras 267 (FCME, UAMIZ).

En los herbarios revisados los ejemplares pertenecientes a la nueva especie se han encontrado determinados con el nombre de Salvia mocinoi Benth. (Fig. 3), la cual presenta: inflorescencias laxas, con entrenudos de más de $1 \mathrm{~cm}$ de largo, brácteas reniformes, acuminadas, con base redondeada o cordada, reflejas o divaricadas, más grandes que los cálices, cálices pubescentes y tubos de la corola ligeramente ventricosos, rectos; mientras que $S$. confertispicata (Fig. 1) tiene inflorescencias compactas, con entrenudos de menos de $1 \mathrm{~cm}$ de largo, brácteas rómbicas, caudadas, con la base cuneada, ascendentes, ligeramente mayores o iguales en tamaño a los cálices, cálices glabrados y corolas con el tubo ventricoso, invaginado (Cuadro 1). 
Fragoso-Martínez y Martínez-Gordillo: Nueva especie de Salvia de Guerrero, México

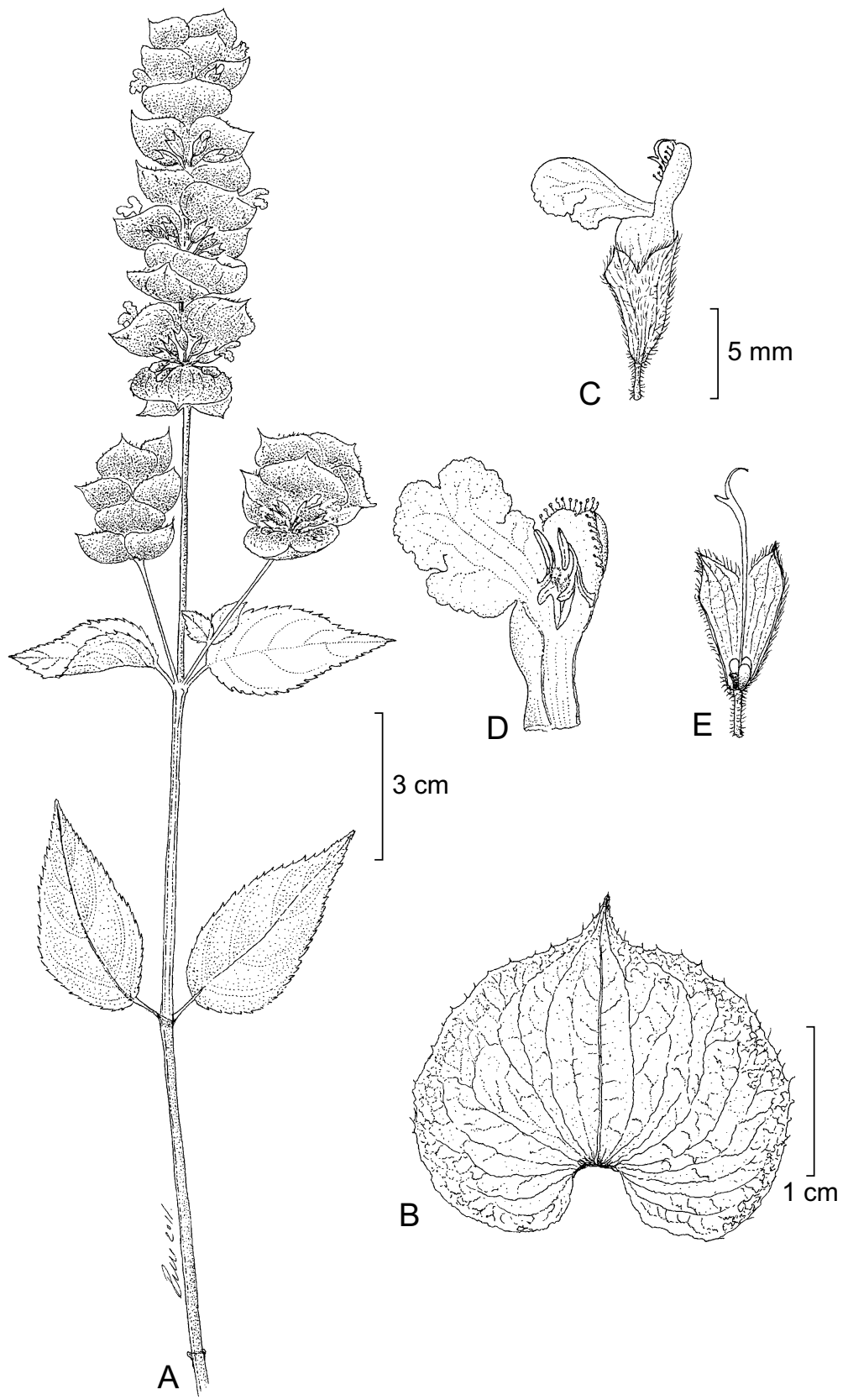

Fig. 3. Salvia mocinoi Benth. A. rama con inflorescencia y hojas; B. bráctea extendida; C. flor; D. corte longitudinal de la corola mostrando el androceo; E. corte longitudinal del cáliz, mostrando el estilo y núculas (Calónico 14030, FCME). 
Cuadro 1. Cuadro comparativo entre Salvia confertispicata y S. mocinoi.

\begin{tabular}{lcc}
\hline & Salvia confertispicata & Salvia mocinoi \\
\hline Haz de las hojas & glabro, excepto en las venas & $\begin{array}{c}\text { esparcidamente hirsuto a } \\
\text { viloso sobre toda la superficie } \\
\text { Tipo de inflorescencia }\end{array}$ \\
Longitud de la inflorescencia & espiga compacta & $3-12(-20) \mathrm{cm}$ \\
Distancia entre verticilastros & $1-4(-6.5) \mathrm{cm}$ & $0.5-2.5(5) \mathrm{cm}$ \\
Forma de las brácteas & $0.3-1 \mathrm{~cm}$ & reniforme \\
Posición de las brácteas & rómbica & divaricadas o reflejas \\
maduras & ascendentes & redondeada o cordada \\
Base de la bráctea & cuneada & acuminado \\
Ápice de la bráctea & caudado & pubescente \\
Pubescencia del cáliz en & glabrado & no invaginado \\
antesis & invaginado & \\
Tubo de la corola & &
\end{tabular}

Se presenta a continuación una clave para identificar las especies de la subsección Lophanthoideae de la sección Membranaceae que crecen en el estado de Guerrero, donde la nueva especie se ha recolectado.

1 Hojas coriáceas; pecíolos menores de $3 \mathrm{~mm}$ de largo ........................ Salvia nitida 1 Hojas membranáceas o papiráceas; pecíolos mayores de $5 \mathrm{~mm}$ de largo.

2 Brácteas ascendentes, con el ápice caudado, inflorescencia compacta, con entrenudos menores de $1 \mathrm{~cm}$ Salvia confertispicata

2 Brácteas reflejas o divaricadas, con el ápice acuminado, inflorescencia laxa, con entrenudos de más de $1 \mathrm{~cm}$.

3 Hojas ovadas; brácteas reniformes; la base redondeada; pedicelos florales erectos; tubo de la corola no invaginado .......................... Salvia mocinoi

3 Hojas ovado-lanceoladas; brácteas deltoide-ovadas, la base cuneada; pedicelos florales reflejos; tubo de la corola invaginado ...... Salvia langlassei

\section{AGRADECIMIENTOS}

Al M. en C. Ramiro Cruz Durán por las ilustraciones. Al Biól. Pablo Lèautaud Valenzuela por el mapa. A Mónica Cardoso por la revisión del resumen en inglés. 
A los curadores de los herbarios CHAP, ENCB, MEXU, UAMIZ y XAL por las facilidades otorgadas en las consultas. A los revisores del manuscrito por sus observaciones.

\section{LITERATURA CITADA}

Bentham, G. 1833. Labiatarum genera et species. Fasc. 3. James Richway and Sons. Londres, Reino Unido. $134 \mathrm{pp}$.

Epling, C. 1939. A revision of Salvia subgenus Calosphace. Repert. Spec. Nov. Regni Veg. 110: $143-153$.

Epling, C. 1940. Supplementary notes on American Labiatae. Bull. Torrey Bot. Club 67(6): 509-534.

Fragoso-Martínez, I. 2011. Revisión taxonómica de la sección Membranaceae del género Salvia en México. Tesis de Licenciatura. Universidad Nacional Autónoma de México. México, D.F., México. 168 pp.

Klitgaard, B. B. 2012. Salvia. In: Davidse, G., M. Sousa S., S. Knapp, F. Chiang y C. Ulloa (eds.). Rubiaceae a Verbenaceae. Vol. 4, núm. 2. Flora Mesoamericana. Missouri Botanical Garden Press. Saint Louis, USA. pp. 147-245.

Ramamoorthy, T. P. y M. Elliot. 1998. Lamiaceae de México: diversidad, distribución, endemismo y evolución. In: Ramamoorthy, T. P., R. Bye, A. Lot. y J. Fa (eds.). Diversidad biológica de México: Orígenes y distribución. Instituto de Biología, Universidad Autónoma de México. México, D.F., México. pp. 501-525.

Villaseñor, J. L. 2003. Diversidad y distribución de las Magnoliophyta de México. Interciencia 28(3): 160-167.

Villaseñor, J. L. 2004. Los géneros de las plantas vasculares de la flora de México. Bol. Soc. Bot. Méx. 75: 105-135. 